\title{
Research of Chinese Stock Market' Complex Network Structure
}

\author{
He Nie ${ }^{1}$, Yuan-Biao Zhang ${ }^{2,3}$, Jun-Ru Chen ${ }^{4} \&$ Xin-Guang Lv $^{2,3}$ \\ ${ }^{1}$ Electrical and Information School, Jinan University, Zhuhai, China \\ ${ }^{2}$ Packaging Engineering Institute, Jinan University, Zhuhai, China \\ ${ }^{3}$ Key Laboratory of Product Packaging and Logistics of Guangdong Higher Education Institutes, Jinan \\ University, Zhuhai, China \\ ${ }^{4}$ International Business School, Jinan University, Zhuhai, China \\ Correspondence: Yuan-Biao Zhang, Packaging Engineering Institute, Jinan University, Zhuhai, 519070, China. \\ E-mail: zybt@jnu.edu.cn
}

Received: March 3, 2015
doi:10.5539/ijef.v7n5p116

Accepted: March 11, 2015

Online Published: April 25, 2015

URL: http://dx.doi.org/10.5539/ijef.v7n5p116

\begin{abstract}
This paper presents and analyzes an analytical model of the stock market's complex network structure, based on the Chinese stock market of the financial industry. Firstly, according to the correlation coefficient formula, we will calculate the correlation coefficient between the stocks by using the stocks data obtained, and we do a quantitative analysis to select the appropriate threshold to filter out low directed correlation numerical value, on this account we establish a stock market's complex network; then using the centrality analysis methods to analyze the stock market's complex network structure, from degree centrality, betweenness centrality, closeness centrality of three aspects to make an analysis, in order to study promoting effects of the leading stocks in the financial industry; and finally we analyze the small-world network characteristics of the stock network established, drawing the conclusion that the small-world network characteristics of the stock network is obvious.
\end{abstract}

Keywords: stock market, complex network, leading stock, centrality analysis, small-world analysis

\section{Introduction}

There is a large number of complex systems can be used to abstract and describe the network (Albert et al., 2002). Complex network theory is the main theory that abstract and describe the complex systems, Zhou et al. (2005) studied the complex network and overview it. Today, this theory was applied more widely in the actual network, covering many areas: Huang et al. (2011) used the complex network method to study the structure of relation between industrial clusters. Through the study of complex networks, scientists have introduced a number of theories, including centrality analysis and the small-world analysis. Ping et al. (2010) used the centrality analysis methods to analyze the micro-blog information dissemination research. Li et al. (2008) studied an empirical study of coauthorship analysis by using social network analysis. Siganos et al. (2003) found that there is a small-world network characteristics in the internet network.

In recent years, it is widely concerned that various phenomena occurring in the stock market has been understood and explained with the concept of the complex networks. Liu et al. (2012) made use of complex network theory to study the stock undirected network topological properties; Ma et al. (2013) studied the complex network relationship between the stock price in order to concluded that there was the correlation between the stock price; Zou (2013) established the undirected network and make use of social network analysis method to study the stock price correlation; Chen (2012) established a composite complex network to study the Chinese stock market network model with dynamics research; Chen (2012) established the stock directed complex network and replied the complex network theory to study the correlation between stocks; Sun (2013) studied stock's price gain and price volatility time series of complex network.

Based on the previous studies, form the point of data mining, quantity statistics, empirical analysis, this paper focuses on establishing the complex network of stock market to study the directed correlation between stocks in the financial industry. In addition, this paper study the structural characteristics of the network by centrality analysis to prove the promoting effect of the leading shares in the financial industry and do an empirical research to prove that the stock network is of small-world network characteristics. 


\section{Stock Market Analysis Model of the Complex Network Structure}

For stock market analysis model of the Complex network structure, Based on the stocks data collected, this paper calculate the stocks' directed correlation numerical value, and reply a threshold to filter low correlation coefficient to establish the complex network of stock market with the rest of the correlation matrix. In order to study the promoting effect of the industry's leading share, using the centrality analysis method to analyze the structure of the network. Finally, this paper conduct a small-world characteristics analysis about the network, and draw conclusions.

\subsection{Establishment of Directed Complex Network Model in Stock Market}

In the stock market, we set a stock a per node and relationship between stocks as edges, therefore the entire stock market can be seen as a complex network with a large number of nodes and edges. In the process of the actual transaction, the interaction relationship between stocks and stocks is directional.

For example, two stocks, $a$ and $b$, in a mutual relationship between them, if price changes of stock $a$ affect $b$ 's price changes, then this effect of relationship is from stock $a$ point to $b$; otherwise from stock $b$ point to $a$. The directed correlation numeral value between each stock can be solved through the following model (Chen, 2012). The following description is specific to the establishment of directed complex network model in stock market :

During the time interval $\Delta t$, return value of a certain stock $a$ at time $t$ is

$$
R_{\Delta t}^{a}(t)=\ln [p(t)]-\ln [p(t-\Delta t)]
$$

Where, $t$ denotes a day, $n$ denotes the number of days, $p(t)$ denotes closing price of a stock at a certain time $t$, $R_{\Delta t}^{a}(t)$ denotes the return value of a stock $a$ 's closing price at time $t$ based on the time interval.

By calculating the matrix $A(t, j)$ about the trading price corresponding to time, you can obtain the matrix $R$ about the price return value of time, that consists of the corresponding each stock's return value $R_{\Delta t}^{a}(t)$.

Do select any two stocks as $a$ and $b$, on the basis of a time interval $\Delta t$, during the entire statistical period, after a time interval $\tau$, the correlation coefficient value of stock $a$ do an impact on stock $b$ is:

$$
Q_{\Delta t}^{a b}(\tau)=\frac{<R_{\Delta t}^{a}(t) R_{\Delta t}^{b}(t+\tau)>-<R_{\Delta}^{a}(t)><R_{\Delta t}^{b}(t+\tau)>}{\sqrt{<\left[R_{\Delta t}^{a}(t)-<R_{\Delta t}^{a}(t)>\right]^{2}>\left[R_{\Delta t}^{b}(t+\tau)-<R_{\Delta t}^{b}(t+\tau)>\right]^{2}>}}
$$

Where, $\langle\ldots\rangle$ denotes the average value, for example, $\left\langle R_{\Delta t}^{a}(t)\right\rangle=\frac{1}{n} \sum_{t=1}^{n} R_{\Delta t}^{a}(t), Q_{\Delta t}^{a b}(\tau)$ represents the correlation coefficient value of stock $a$ do an impact on stock $b$, after a time interval $\tau$, on the basis of a time interval $\Delta t$.

The directed correlation numeral value between the two stocks between -1 and 1 , that is, $-1<Q_{\Delta t}^{a b}(\tau)<1$. If, $0<Q_{\Delta t}^{a b}(\tau)<1$ then stock $a$ and $b$ are positively correlated, that is, the price fluctuation of stock $a$ will affect stock $b$ toward the same direction fluctuations; otherwise it is stock $a$ and $b$ are negatively correlated; if, $Q_{\Delta t}^{a b}(\tau)=0$ then stock $a$ and stock $b$ have no correlation and will not affect each other. And also, if, $\left|Q_{\Delta t}^{a b}(\tau)\right|<\left|Q_{\Delta t}^{b a}(\tau)\right|$ then that relationship interaction flows between stock $a$ and $\mathrm{b}$ is from $b$ to $a$, that is, in the network graph, there exists the directed edge from stock $b$ point to $a$; If, $\left|Q_{\Delta t}^{a b}(\tau)\right|>\left|Q_{\Delta t}^{b a}(\tau)\right|$, then that relationship interaction flows between stock $a$ and $\mathrm{b}$ is from $a$ to $b$, that is, in the network graph, there exists the directed edge from stock $a$ point to $b$; if, $\left|Q_{\Delta t}^{a b}(\tau)\right|=\left|Q_{\Delta t}^{b a}(\tau)\right|$ then it is considered the interaction between the two stocks $a$ and $b$ is equal, no relationship interaction. that is, in the network graph, there is no edge from stock $a$ and $b$.

Thus, directed complex network model in stock market can be built out.

\subsection{Structural Analysis of Complex Networks}

\subsubsection{Three Methods of Centrality Analysis}

Centrality analysis is one of the key analyses in complex network analysis. Centrality analysis can determine the specific position of stocks in the complex network of stock market built. According to different algorithms, the centrality can be divided into three types. 
(1) Degree centrality. In the stock market network, if there are correlations between a stock and many other stocks, then the stock have a concentrated status in the network, and it owns a strong importance. Under the guidance of this idea, the degree centrality of the network can be measured by the number of nodes by another node connected with each other in the network.

(2) Betweenness centrality. In the stock market network, if a stock is located in the route connected with other stocks, we hold the opinion that it is located in an important location, because it can control the contact with the other two stocks. According to the idea, betweenness centrality can measure the ability of the stock's resource control. If a stock is on many other' shortest route, it has a high betweenness centrality.

(3) Closeness centrality. In the stock market network, closeness centrality explains the importance of stock's transinformation. When closeness centrality is small, indicating that the relationship between the stocks and other stocks is close; when closeness centrality is small, indicating that the relationship between the stocks and other stocks is loose.

\subsubsection{Small-World Characteristic Analysis}

In different complex networks, the average path length has its different meanings. This paper mainly refers to the average value of stock's correlation numeral value. If the scale of a complex network and the number of nodes are large, but the average path length of the network is very small, it is said that the network has small world effect, with qualitative language can be described as follows: as the average degree $<k>$ of nodes, the network path length $L$, the growth rate of them, at most, proportional to the logarithms of nodes' number $N$ in network.

Another measure indicator to the characterization of small-world is the clustering coefficient, clustering coefficient $C$ is used to describe the aggregation of nodes in the network. If the research network has a smaller characteristic path length and a larger clustering coefficient, it is considered that the network is small-world network. The smaller and larger here all compare a random network as a reference object.

$L_{\text {randam }}$ is characteristic path length of random networks, if random network and the network researched have the same number of nodes and edges (Liu Xiao-xia, 2012). It can be expressed as:

$$
\begin{gathered}
L \geq L_{\text {random }} \quad C>C_{\text {random }} \\
L_{\text {random }}=\frac{\ln n}{\ln k} \\
C_{\text {random }}=\frac{k}{n}
\end{gathered}
$$

Where, $k$ denotes average degree of the network, in general, is $\frac{2 Q}{n}$ ( $Q$ is the number of edges of network).

\section{Instance Analysis}

\subsection{Collection and Collation of Data}

Firstly, with RESSET database query system (http:/202.204.164.18/), this paper filter out all closing prices of trading days of all the financial industry stocks listed on the Shanghai Stock Exchange and Shenzhen Stock Exchange form June 30, 2012 to June 30, 2014. In order to analyze the data, organizing data with specific rules: If there is a trading price of missing data situation and then delete all the stock price information about this trading day. And delete missing seriously stock data. After a number of unified data collations, finally getting 36 stocks with trading days' closing price data in financial industry between June 30, 2012 to June 30, 2014, they are: Ping An Bank Co., Ltd (000001), Shaanxi International Trust Co., Ltd (000563), Northeast Securities Co., Ltd (000686), Guo Yuan Securities Co., Ltd (000728), GF Securities Co., Ltd (000776), Chang Jiang Securities Co., Ltd (000783), Bank of Ningbo (002142), Western Securities Co., Ltd (0002673), Shaihang Pudong Development Bank (600000), Huxia Bank Co., Ltd (600015), China Minsheng Banking Co., Ltd (600016), Cittc Securities Co., Ltd (600030), China Merchants Bank (600036), Southwest Securities Co., Ltd (600369), Shanghai AJ Co., Ltd (600643), Haitong Securities Co., Ltd (600837), China Merchants Securities (600999), Bank of Nanjing (601009), CIPC (601099), Industrial Bank Co., Ltd (601166), Bank of Beijing Co., Ltd (601169), Agicultural Bank of China (601288), Ping An Insurance (Group) Company Of China, Ltd (601318), Bank of Communications Co., Ltd (601378), New China Life Insurance Company Ltd. (601336), Industrial Securities Co., Ltd (601377), Industrial and Commercial Bank of China (601398), Soochow Securities Co., Ltd (601555), China Pacific Insurance (GROUP) Co., Ltd (601601), China Life Co., Ltd (601628), Huatai Securities 
Co., Ltd (601688), Everbright Securities Co., Ltd (601788), China Everbright Bank Co., Ltd (601818), China Construction Bank Co., Ltd (601939), Bank of China Co., Ltd (601988), China CITIC Bank Co., Ltd (601998). Because of the limited space of this paper, all the following analysis use stock's number on behalf of the stock.

\subsection{Construction of Financial Chinese Stocks' Directed Complex Network}

We use the directed complex network model, and we calculate the directed correlation coefficient between 36 stocks in financial industry by MATLAB programming, and we obtain the correlation coefficient matrix. Based on this process, the distribution of directed correlation numerical value is shown in Figure 2. Based on the study of the financial industry stocks correlation in the stock market, that is the strength of links between stocks. To prominent the strong correlation nodes in the network, the paper filtered out the stocks that price fluctuations have little effect on network by setting the threshold $\delta$. (The method is that the absolute value of the directed correlation numerical value is less than the threshold value, set to 0 .) It means that under certain conditions, the correlation between some of the stocks does not exist. Filtering out the correlation coefficient changes the density of edge and the degree of nodes, so that the relevance of the network is more obvious, and it is easy to analyze. If there is no threshold, the nodes of network will be messy as well as the directed network diagram.

From the correlation distribution of Figure 1, in the non-set threshold, the correlation coefficient is a non-zero constant between -0.2 to 0.25 . In this paper, setting 0.02 as the threshold change unit to filter and statistics the correlation coefficients under the different thresholds. Found that with the increase of the threshold, the remaining correlation coefficients become less and less, the strong correlation between stocks becomes more obvious. When the threshold is large enough, the only one node is remaining. For example, when the threshold is 0.03 , correlation matrix remains 420 coefficients; when the threshold is 0.13 , the matrix is leaving only 19 coefficients; when the threshold is 0.21 , only a coefficient is remaining. The variation curve of correlation coefficient with the change of the threshold is shown in Figure 2.

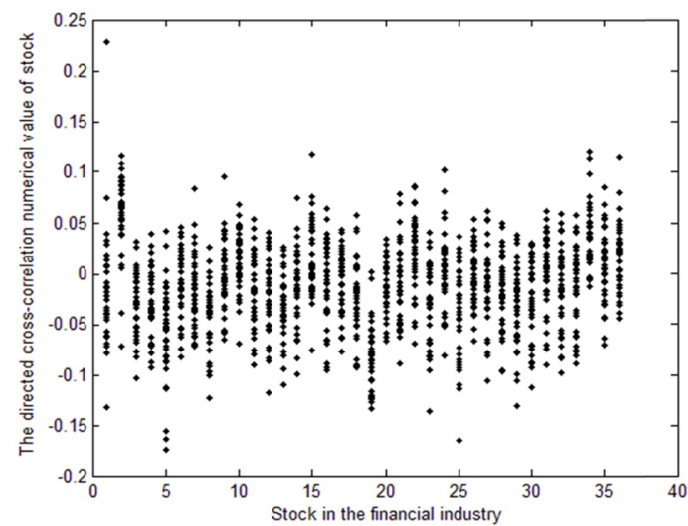

Figure 1. The distribution of correlation coefficient

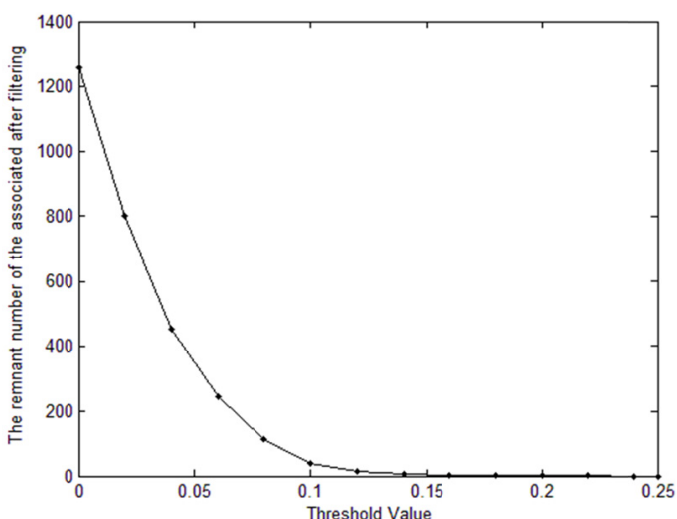

Figure 2. The distribution of the correlation coefficient's number 
From the curve in Figure 2, with increase of the threshold, the number of correlation coefficients decrease faster and faster. And when the threshold is 0.1 , the slope of curve is max. This means that with the threshold increases, the number of the large correlation coefficient become less. Thus, there is a small number of stocks that have a greater impact in financial industry.

According to the curve, it is found that when the threshold is about 0.1 , slope of curve is the largest. Thus this paper set 0.1 as the threshold to filter out the correlation coefficient matrix and convert into UCINET data, then obtain the financial industry stocks' directed complex network diagram. In this directed network, each of the nodes represent a financial stocks, the attachment is showing that there is an correlation between them. After the threshold setting, the directionality of the network is obvious, showing that some nodes have strong ties, which indicate that the stock price change lead to price fluctuations along with its all associated stocks. And ultimately affect the whole trend of the financial industry. Directed network diagram can be built in this way, which is shown in Figure 3 below.

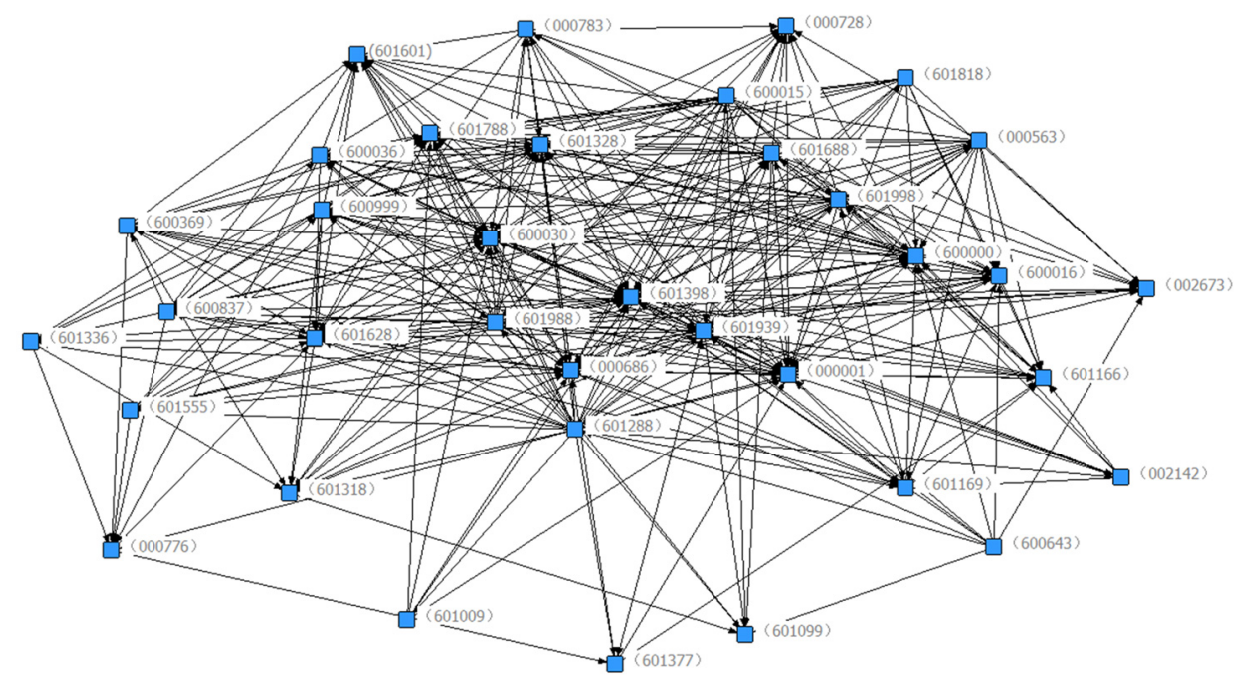

Figure 3. Stocks' network diagram

In Figure 3, the entire network shows comparison regular state. There is a stronger link between the nodes. The figure clearly show that there is not scatter in the network. Each node is connected to other nodes, the network can be applies to the centrality analysis in the following section.

\subsection{Centrality Analysis}

Centrality Analysis has been one of the important part of the network analysis. Network centricity is of three indicators, respectively is the degree centrality, betweenness centrality, closeness centrality. This section analyzes the network built comprehensively through the three indicators.

\subsubsection{Degree Centrality}

The degree of the nodes in the stocks market' network means this node related to other nodes in the stock market. The greater the value, the more the relationship between nodes and other nodes. If the value is big, the nodes in the network are also in the intensive position, otherwise loose. In this paper, the filtered correlation coefficients by threshold of 0.1 are analyzed, and some specific indices of the optimal results in following Table 1.

Table 1. Some optimal result of degree centrality

\begin{tabular}{cccc}
\hline Stocks' num & Degree & NrmDegree & Share \\
\hline$(601288)$ & 2.309 & 28.943 & 0.139 \\
$(601939)$ & 1.783 & 22.347 & 0.107 \\
$(601398)$ & 1.729 & 21.674 & 0.104 \\
$(600030)$ & 1.069 & 13.395 & 0.064 \\
$(601988)$ & 1.046 & 13.116 & 0.063 \\
$(000686)$ & 1.018 & 12.766 & 0.061 \\
\hline
\end{tabular}


Table 1 shows some partial results of data analysis in the financial industry, the largest degree centrality is 601288 , followed by $601939,601398,600030,601988,000686$. The degree centrality value is far greater than the average of the degree (0.462). In terms of the entire financial industry directed network, degree of 601288 is the largest, and its relative degree of centrality and relationships share is far greater than other stocks. In the entire network, 601288 is in the denser areas, it occupies a central position. In all nodes, just 6 nodes' degrees are greater than 1 , indicating the number of shares has influence in the financial industry is small. Just a small part of the stocks' price fluctuations lead the whole industry's stock's price volatility.

\subsubsection{Betweenness Centrality}

In the stock market, degree of betweenness centrality reflects promoting effect between the stocks and some stocks for the degree of resource information control. In this paper, the filtered correlation coefficients by threshold of 0.1 are analyzed, and some specific indices of the optimal results in following Table 2.

Table 2. Some optimal result of betweenness centrality

\begin{tabular}{ccc}
\hline Stocks' num & Betweenness & nBetweenness \\
\hline$(601939)$ & 303.745 & 25.525 \\
$(601398)$ & 278.666 & 23.417 \\
$(000001)$ & 90.379 & 7.595 \\
$(601988)$ & 60.370 & 5.073 \\
$(600036)$ & 58.647 & 4.928 \\
$(601328)$ & 48.711 & 4.093 \\
\hline
\end{tabular}

From Table 2, the indices of 601939 and 601398 in the betweenness centrality are far more than other stocks. It indicates that these two stocks are on the shortest way to other stocks in the entire financial industry network. We take the results in section 3.3.1 into consideration, 601939 and 601398 in the dense and central location of the entire network, there are many relationships they build with others, and the same times the dependence of the other nodes to them is highest, indicating that they are in the central position of the entire network. The third is 000001 , relative to other stocks, its value of betweenness centrality is high. It plays a less vital role on dependent on the extent of the network and the degree of control in the network.

From the financial industry stocks studied in this paper, the maximum of the betweenness centrality is 303.745 , the minimum value is 0 . The sixth of betweenness centrality is 601328 , and its value is 48.711 . And there are 4 stocks with 0 degree. So, there are a few stocks in the financial industry can master resources control ability.

\subsubsection{Closeness Centrality}

Closeness centrality measure the degree of a node is not controlled by other nodes. Contrary to the above two central degrees, the value is the small, explaining that the node is at the core position. In this paper, the filtered correlation coefficients by threshold of 0.1 are analyzed, and some optimal indices of the optimal results in following table3.

Table 3. Some optimal result of farness centrality

\begin{tabular}{ccccc}
\hline Stocks'num & inFarness & outFarness & inCloseness & outCloseness \\
\hline$(601398)$ & 74.000 & 94.000 & 47.297 & 37.234 \\
$(600030)$ & 78.000 & 111.000 & 44.872 & 31.532 \\
$(000001)$ & 79.000 & 99.000 & 44.304 & 35.354 \\
$(601988)$ & 84.000 & 99.000 & 44.304 & 35.354 \\
$(601628)$ & 85.000 & 113.000 & 41.176 & 30.973 \\
$(601328)$ & 85.000 & 103.000 & 41.176 & 33.981 \\
\hline
\end{tabular}

The results can be seen form Table 3, the degree of closeness centrality in the top position is smaller, the centrality is higher, the closer the distance between the node and other nodes, the closer the distance information to all other nodes also. The distance of 601939 information reach all the nodes is smallest with 74 steps, the distance of obtaining information is 94 steps. It is less than other stocks. 601398 is in the core position in the network ,it is easy to pass information to other stocks and obtain the information from other stocks. The forefront of the stocks as well as $600030,000001,601988,601628,601328$, they are also in an important position in the 
network. And its independence is strong, not easy to be controlled. Relatively speaking, low-ranking stocks in the analysis, require the greater distance to obtain the information and transfer the information, the more dependence on the network to other stocks. It is easier to under the control of the other stocks. When the stock's price changes or significant information affecting, the independence weaker stock's price change accordingly.

\subsubsection{Correspond to the Real Economy Analysis of the Financial Industry}

This section compares the financial industry stocks with its corresponding listed companies, to verify the applicability of the directed network correlation model.

Section 3.3.1, 3.3.2 and 3.3.3 show that, in this research, the greater degree of influence of the financial industry stocks is $601939,601398,000001,601288,601988,601328$. In order to contact the actual situation to analyze strength of the relationship between the financial industry stocks, we collect total profits of some listed companies studied from the annual report at the end of 2013. The part of the total profit of some listed companies in the financial industry at the end of 2013 is shown in Table 4.

Table 4. Total profits of some listed companies in financial industry by the end of 2013

\begin{tabular}{cccc}
\hline Stocks'num & Companies' name & profit(yuan) & Ranking \\
\hline 601398 & Industrial And Commercial Bank Of China & 338537000000.00 & 1 \\
601939 & China Construction Bank & 279806000000.00 & 2 \\
601288 & Agricultural Bank of China & 214174000000.00 & 3 \\
601988 & Bank Of China & 212777000000.00 & 4 \\
601328 & Bank of Communications & 79909000000.00 & 5 \\
601318 & Ping An Insurance (Group) Company Of China & 46224000000.00 & 11 \\
\hline
\end{tabular}

In the Table 4, the total profit at the end of 2013 rank the forefront is Industrial and Commercial Bank of China, China Construction Bank, Agricultural Bank of China, Bank of China, Bank of Communications. Combined with the centrality analysis, finding that, in the financial industry, the more active in the stock, the more central position the stocks are located in the network, and the stronger the ability to control the resources of other stocks, the greater influence of those stocks' price change. Besides, the total profits of those stocks corresponding listed companies in financial industry is relatively high. The companies occupies a core position in the industry. Those companies are the industry's leading enterprises, the corresponding stocks are the leading stocks.

\subsection{Small-World Analysis}

If a network is with small world effect, the network is a smooth information network. If industry's directed network is with small-world effect, the stocks in the network can smoothly communicate with each other. The price change information can be quickly transferred to others, causing price changes in other stocks.

In this paper, the filtered correlation coefficients by threshold of 0.1 are analyzed, the average distance to the stock network is 1.961, the average distance of such random network (according to the calculation method of section 2.2.3) is 0.553 , shows that the average stock network distance is greater than the average distance of random networks. The stock network clustering coefficient is 0.435 , and the network of the random network clustering coefficient is 0.288 . The stock of network clustering coefficient is greater than the clustering coefficient of random networks. According to the section 2.2.3, therefore the stock show obvious small-world network characteristics. A further analysis of the network from the network size frequency is made, and get the results in the following table 5.

Table 5. Statistical description of the stocks' network

\begin{tabular}{lll}
\hline \multicolumn{3}{l}{ Frequen Proport } \\
\hline 1 & 374.000 & 0.314 \\
2 & 520.000 & 0.437 \\
3 & 267.000 & 0.224 \\
4 & 29.000 & 0.024 \\
5 & 1.000 & 0.001 \\
\hline
\end{tabular}


Table 5 shows that distance 1 had 374 times, accounting for $31.4 \%$ of the total, from 2 had 520 times, accounting for $43.7 \%$ of the total, from 3 had 267 times, $22.4 \%$ of the total. In this 36 stocks, the maximum value is 5 , the minimum value is 1 , and the average distance is 1.961 . It Shows that in the financial industry stocks, every two stocks can establish contact with each other by 1.961 nodes. According to the theory of small-world effect principle: generally the distance not more than 10 networks can be said to have a small-world effect (Zou, 2013). It describes the financial industry stocks have a strong connection to the network , as well as having the ability to respond quickly and high efficiency.

\section{Conclusion}

This paper studies the stock market complex networks of the financial industry, and proposes a stock market analysis model of the complex network structure.

First, we take into account the directed correlation between stocks, and then according to correlation coefficient formula of stocks, we calculate the stock data obtained to get correlation coefficient between stocks. We do a numerical analysis combine with coefficient distribution figure to determine the optimal threshold to filter coefficients of the stock relationship in the financial industry, on this account we establish a strong directed correlation complex network.

Then, in order to determine the status of specific stocks in the complex network of the stock market, we use centrality analysis to analyze the stock complex network. From degree centrality, betweenness centrality, and closeness centrality of three aspects to have a network analysis, we obtain stocks with higher status. And then we combine with the profit value of stocks of the corresponding listed companies, in order to study the leading role in the financial industry, finding that stocks have higher status often have higher profit value.

Finally, we analyze small-world network characteristics of complex networks established, form the average distance of the network, and the distance frequency analysis between the network to analyze, making the conclusion that the small-world characteristics of stock network is obvious.

The research methods is feasible in actual life. For the financial stock market analysis, in the further research, we can use the complex network theory to predict the price of the financial markets, and the optimal portfolio, etc.

\section{Acknowledgements}

The authors acknowledge the financial support of this research by the Key Laboratory of Product Packaging and Logistics of Guangdong Higher Education Institutes, the Fundamental Research Funds for the Central Universities, the project of the Natural Science Foundation of Guangdong Province (No.S2012010008773), the projects of Zhuhai Science, Technology, Industry, Trade and Information Technology Bureau (No.2011B050102013 \& 2012D0501990033), the project of the college students' innovation and entrepreneurship training plan in Jinan university (national level) (No. 201410559004).

\section{References}

Albert, R., \& Barabsi, A. (2002). Statistical mechanics of complex networks. Rev Mod Phys, 74(1), 47-97. http://dx.doi.org/10.1103/RevModPhys.74.47

Chen, H. (2012). Directed Cross-correlation Research Between Stocks Based on Complex N-etwork. Beijing University of Posts and Telecommunications.

Chen, J. H. (2012). Dynamic Study on Network Models of Chinese Stock Market. Zhejiang University of Technology.

Huang, Z. H., Lu, J., Sun, H. P., Hu, J. F., \& Song, Y. (2011). Sticky factors in the industrial relocation of a cluster: A case study of Zhili children's garments cluster in China. The Social Science Journal, 48(3), 560-565. http://dx.doi.org/10.1016/j.soscij.2011.07.003

Li, L., \& Zhu, Q. H. (2008). An Empirical Study of Coauthorship Analysis Using Social Network Analysis. Information Science, 4, 549-555.

Liu, X. X., \& Wang, W. H. (2012). Characterisitic Analysis of Complex Network for Shanghai Stock Market. Journal of WUT, 5, 642-645.

Ma, L. G., \& Sun, R. C. (2013). Shang hai Stock network-based multi-subnet Composite Complex Network Characterisitics. Science \& Technology Information, 9, 132-133.

Ping, L., \& Zong, L. Y. (2010). Research on Microblog Information Dissemination Based on SNA Centrality Analysis-A Case Study with Sina Microblog. Books of Intelligence Knowledge, 6, 92-97. 
Siganos, G., Faloutsosos, M., \& Faloutsosoc. (2003). Power laws and the AS level internet topology. IEEE/ACM Transaction on Networking, 11(4), 514-524. http://dx.doi.org/10.1109/TNET.2003.815300

Sun, J. (2013). Characterization of Price Return and Volatility Time Series by Complex Ne-tworks. Harbin Institute of Technology.

Zhang, D., \& Zhuang, X. D. (2011). Analyzing Characteristics of the Complex Network of Stock Risk During Unilateral Decline of the Shanghai Stock Market. Journal of Northeastern University (Natural Science), 4, 604-608.

Zhang, L. H. (2013). Simulation and Application Research on Complex Networks Modeling. Dalian University of Technology.

Zhou, T., Bai, W. C., Wang, B. H., Liu, Z. J., \& Yan, G. A. (2005). Brief review of complex networks. Physics, 1, 31-36.

Zhuang, X. T., Min, Z. F., \& Chen, S. Y. (2007). Characteristic Analysis of Complex N-etwork for Shanghai Stock Market. Journal of Northeastern University (Natural Science), 7, 1053-1056.

Zou, C. (2013). The Social Network Analysis of the ShangHai Stock Market Based on the Correlation Coefficient of the Stock Price. Beijing University of Technology.

\section{Copyrights}

Copyright for this article is retained by the author(s), with first publication rights granted to the journal.

This is an open-access article distributed under the terms and conditions of the Creative Commons Attribution license (http://creativecommons.org/licenses/by/3.0/). 